\title{
The impact of COVID-19 on rare metabolic patients and healthcare providers: results from two MetabERN surveys
}

\author{
C. Lampe ${ }^{1}$, C. Dionisi-Vici², C. M. Bellettato ${ }^{3}$, L. Paneghetti ${ }^{3}$, C. van Lingen ${ }^{3}$, S. Bond ${ }^{3}$, C. Brown ${ }^{4}$, A. Finglas ${ }^{5}$, \\ R. Francisco ${ }^{6}$, S. Sestini' ${ }^{7}$ J. M. Heard ${ }^{3}$ and M. Scarpa ${ }^{3^{*}}$ (DMetabERN collaboration group
}

\begin{abstract}
The ongoing coronavirus disease 2019 (COVID-19) pandemic has caused disruption in all aspects of daily life, including the management and treatment of rare inherited metabolic disorders (IMDs). To perform a preliminary assessment of the incidence of COVID-19 in IMD patients and the impact of the coronavirus emergency on the rare metabolic community between March and April 2020, the European Reference Network for Hereditary Metabolic Diseases (MetabERN) has performed two surveys: one directed to patients' organizations (PO) and one directed to healthcare providers (HCPs). The COVID-19 incidence in the population of rare metabolic patients was lower than that of the general European population $(72.9 \times 100,000$ vs. $117 \times 100,000)$. However, patients experienced extensive disruption of care, with the majority of appointments and treatments cancelled, reduced, or postponed. Almost all HCPs (90\%) were able to substitute face-to-face visits with telemedicine, about half of patients facing treatment changes switched from hospital to home therapy, and a quarter reported difficulties in getting their medicines. During the first weeks of emergency, when patients and families lacked relevant information, most HCPs contacted their patients to provide them with support and information. Since IMD patients require constant follow-up and treatment adjustments to control their disease and avoid degradation of their condition, the results of our surveys are relevant for national health systems in order to ensure appropriate care for IMD patients. They highlight strong links in an interconnected community of HCPs and PO, who are able to work quickly and effectively together to support and protect fragile persons during crisis. However, additional studies are needed to better appreciate the actual impact of COVID-19 on IMD patients' health and the mid- and long-term effects of the pandemic on their wellbeing.
\end{abstract}

Keywords: COVID-19, SARS-CoV-2, Coronavirus, Pandemic, Rare diseases, Inherited metabolic diseases, IMD, Survey

\section{Background}

Coronavirus disease 2019 (COVID-19) is a novel infectious disease caused by the severe acute respiratory syndrome coronavirus 2 (SARS-CoV-2), which emerged in China in December 2019. The new coronavirus spread rapidly across the globe, and on 11th March 2020 the World Health Organization (WHO) classified the

\footnotetext{
*Correspondence: maurizio.scarpa@metab.ern-net.eu

${ }^{3}$ MetabERN, Regional Coordinating Center for Rare Diseases, Udine University Hospital, Udine, Italy

Full list of author information is available at the end of the article
}

outbreak as a pandemic [1]. As of 20th October 2020 there have been 40,114,293 confirmed cases of COVID19 worldwide-of which 8,027,954 in Europe-and $1,114,692$ global deaths [2], with different incidence rates and fatalities between countries and regions. Since the pandemic is still ongoing, these numbers are not definitive and, unfortunately, by the time this paper is published the numbers will be higher.

Compared to other coronaviruses such as SARS-1 and MERS, to date SARS-CoV-2 has showed a lower fatality rate (2.3\% vs. $9.5 \%$ and $34.4 \%$, respectively) [3]. The presence of underlying health conditions, such as 
diabetes, hypertension and cardiovascular disease, have been correlated with severe COVID-19 and death [4].

The ongoing pandemic of SARS-CoV-2 has suddenly changed all aspects of our daily lives, including how healthcare is organized and delivered. Indeed, the pandemic has severely affected national health systems across Europe: besides the immediate strain put on intensive care units and medical professionals assisting those with COVID-19, most hospitals had to cancel and/or postpone most non-urgent visits, procedures, and therapies, adapting the type and modality of care that they provide to patients affected by all diseases, including those with rare inherited metabolic diseases (IMDs).

IMD patients require constant follow-up and adjustments of treatment and diet, as metabolic destabilization may happen in case of events such as fever or infection. In ordinary situations, IMDs are neglected conditions, with limited resources and attention dedicated to these rare diseases. The COVID-19 pandemic has reduced attention even further. Disruption of the proper followup of IMD patients can drastically compromise their clinical condition and aggravate the course of the disease, not only from a medical, but also from a social and psychological point of view, with the possible onset of depression, anxiety, and panic attacks.

Recent surveys from international patients organizations (PO) such as EURORDIS [5] and NORD [6] have revealed the detrimental effects of SARS-CoV-2 on the rare disease community worldwide: from interruption of care and closed hospitals, to medication shortages and significant concern regarding COVID-19 and its impact on the present and future management of rare diseases. Similar results have been observed in national studies conducted in Italy [7] and Ireland [8]. These results show that, in the current emergency situation, surveys are important resources to understand current and future needs of rare disease patients. This information is indeed crucial to organize an appropriate response and to prioritize actions of healthcare providers (HCPs) in the coming months, with the aim of minimizing COVID-19 impact on rare disease patients.

Besides the aforementioned PO, additional surveys have been activated by European Reference Networks (ERNs) to gather more details on the effects of the coronavirus pandemic on specific groups of rare disease patients. Among these, the ERN for Hereditary Metabolic Diseases (MetabERN) has performed two surveys to understand the situation in the rare metabolic community. MetabERN connects centers specialized in rare metabolic diseases at EU level; it represents 77 HCPs from 23 EU Member States and 44 PO, and it is endorsed by the Society for the Inborn Errors of Metabolism (SSIEM). As such, MetabERN is the main reference point and cluster of expertise for the rare metabolic community in Europe.

While information from the patients' perspective is crucial to understand their unmet medical needs and find appropriate solutions to guarantee their wellbeing, gathering data from HCPs is equally important to have a more comprehensive view of the rare disease community during the ongoing coronavirus emergency. Indeed, HCPs can provide information on the most effective and successful measures that have been implemented to deliver medical care during the pandemic, and can also provide data on the infection rate and the clinical manifestations of SARS-CoV-2 infection in rare disease patients. However, no surveys regarding COVID-19 are currently available from the HCPs point of view.

Here we present the first surveys performed in the field of rare metabolic diseases to analyse the impact of the coronavirus pandemic on the whole metabolic rare disease community: one survey was directed to IMD PO and one was specific for HCPs.

\section{Methods}

The surveys' design and data collection were performed using the Survey Monkey platform. The survey for HCP (Additional file 1 in the Supplementary Information) consisted of 30 questions and was distributed to all 77 MetabERN centres at the start of the emergency, between 29th March and 17th April 2020. The survey for PO (Additional file 2 in the Supplementary Information) included 32 questions, was active at the beginning of the pandemic, between 26th March and 14th April 2020, and was sent to 45 associations. All data were extracted and analysed using Microsoft Excel. Epidemiological analysis was performed by integrating the survey's data with the MetabERN database (number of adult and paediatric patients followed by each HCP member of the MetabERN). All participants gave their consent for data collection and publication.

\section{Results \\ HCP survey \\ Incidence of COVID-19 in rare metabolic patients}

The results of the HCPs survey were collected from 73 MetabERN units in 21 EU Member States, with a response rate of 95\%. Most of these centers (64.4\%) assist both adult and paediatric patients, about a quarter $(24.7 \%)$ provide only paediatric care, and $11 \%$ follow adult patients (Table 1).

As of 17th April 2020, only 13 paediatric cases of COVID-19 were registered in just 7\% of centres: at the time of diagnosis and also during the course of infection, 12 patients showed mild symptoms of the disease, while one patient was asymptomatic (Table 1). These paediatric 
Table 1 Responses collected from the HCPs survey

\begin{tabular}{|c|c|}
\hline Category & $n(\%)$ \\
\hline \multicolumn{2}{|l|}{ Group of IMD patients followed at the centre } \\
\hline Adult & $8 / 73(11)$ \\
\hline Paediatric & $18 / 73(24.7)$ \\
\hline Both & $47 / 73(64.4)$ \\
\hline \multicolumn{2}{|c|}{ Paediatric patients infected with SARS-CoV-2 (confirmed by testing) in the centre } \\
\hline Yes & $5 / 73(6.9)$ \\
\hline No & $62 / 73(84.9)$ \\
\hline Do not know & $6 / 73(8.2)$ \\
\hline Total number of paediatric reported cases & 13 \\
\hline \multicolumn{2}{|l|}{ Symptoms of paediatric patients positive for COVID-19 } \\
\hline \multicolumn{2}{|l|}{ At diagnosis } \\
\hline Asymptomatic & $1 / 13(8)$ \\
\hline Mild & $12 / 13(92)$ \\
\hline Severe & $0 / 13(0)$ \\
\hline Do not know & $0 / 13(0)$ \\
\hline \multicolumn{2}{|l|}{ During the infection } \\
\hline Asymptomatic & $1 / 13(8)$ \\
\hline Mild & $12 / 13(92)$ \\
\hline Severe with need of hospitalisation & $0 / 13(0)$ \\
\hline Required intensive care & $0 / 13(0)$ \\
\hline \multicolumn{2}{|c|}{ Adult patients infected with SARS-CoV-2 (confirmed by testing) in the centre } \\
\hline Yes & $7 / 73(9.6)$ \\
\hline No & $54 / 73(74)$ \\
\hline Do not know & $12 / 73(16.4)$ \\
\hline Total number of adult reported cases & 11 \\
\hline \multicolumn{2}{|l|}{ Symptoms of adult patients positive for COVID-19 } \\
\hline \multicolumn{2}{|l|}{ At diagnosis } \\
\hline Asymptomatic & $0 / 11(0)$ \\
\hline Mild & 10/11 (91) \\
\hline Severe & $1 / 11(9)$ \\
\hline Do not know & $0 / 11(0)$ \\
\hline \multicolumn{2}{|l|}{ During the infection } \\
\hline Asymptomatic & $0 / 11(0)$ \\
\hline Mild & 9/11 (82) \\
\hline Severe with need of hospitalisation & $2 / 11(18)$ \\
\hline Required intensive care & $0 / 11(0)$ \\
\hline \multicolumn{2}{|l|}{ Casualties due to COVID-19 among IMD patients } \\
\hline Yes & $0 / 73(0)$ \\
\hline No & $64 / 73(87.7)$ \\
\hline Do not know & 9/73 (12.3) \\
\hline \multicolumn{2}{|l|}{ Change needed in the management of IMD patients } \\
\hline Yes & $66 / 73(90.4)$ \\
\hline No & $7 / 73(9.6)$ \\
\hline \multicolumn{2}{|c|}{ Change needed in the therapy regime of IMD patients with no COVID-19* } \\
\hline Yes, the frequency of therapy has been reduced & 19/73 (26) \\
\hline Yes, the therapy has been stopped & $3 / 73(4.1)$ \\
\hline Yes, the frequency of rehabilitation has been reduced & $12 / 73(16.4)$ \\
\hline Yes, rehabilitation has been stopped & 15/73 (20.6) \\
\hline Only for some specific cases & $14 / 73(19.2)$ \\
\hline No & 26/73 (35.6) \\
\hline \multicolumn{2}{|c|}{ Changes in therapeutic regimes unified at national or regional level } \\
\hline Yes & $25 / 45(55.6)$ \\
\hline No & $20 / 45(44.4)$ \\
\hline
\end{tabular}


Table 1 (continued)

\begin{tabular}{|c|c|}
\hline Category & n (\%) \\
\hline \multicolumn{2}{|c|}{ Proportion of missed outpatient visit for IMD at the centre } \\
\hline $0-25 \%$ & $5 / 73(6.9)$ \\
\hline $25-50 \%$ & $3 / 73(4.1)$ \\
\hline $50-75 \%$ & 20/73 (27.4) \\
\hline $75-100 \%$ & $40 / 73(54.8)$ \\
\hline Not applicable & $5 / 73(6.9)$ \\
\hline \multicolumn{2}{|c|}{ Outpatient face-to-face visits replaced by video conference/telephone interaction } \\
\hline Yes & $66 / 73(90.4)$ \\
\hline No & $4 / 73(5.5)$ \\
\hline Not applicable & $3 / 73(4.1)$ \\
\hline \multicolumn{2}{|c|}{ Patients stopped treatment by their own decision } \\
\hline Yes & $10 / 73(13.7)$ \\
\hline No & $57 / 73(78.1)$ \\
\hline Do not know & $6 / 73(8.2)$ \\
\hline \multicolumn{2}{|c|}{ Disease categories expected to be at major risk in relation to COVID-19* } \\
\hline $\mathrm{AOA}$ & $42 / 73(57.5)$ \\
\hline PM-MD & $41 / 73(56.2)$ \\
\hline C-FAO & $33 / 73(45.2)$ \\
\hline LSD & $38 / 73(52)$ \\
\hline PD & 14/73 (19.2) \\
\hline CDG & $18 / 73(24.7)$ \\
\hline NOMS & $9 / 73(12.3)$ \\
\hline \multicolumn{2}{|c|}{ Awareness of good-quality informative material about COVID-19 and IMD } \\
\hline Yes & 26/73 (35.6) \\
\hline No & $33 / 73(45.2)$ \\
\hline Do not know & $14 / 73(19.2)$ \\
\hline \multicolumn{2}{|c|}{ Centre produced informative material about COVID-19 and IMD } \\
\hline Yes & $27 / 73(37)$ \\
\hline No & $42 / 73(57.5)$ \\
\hline Do not know & $4 / 73(5.5)$ \\
\hline \multicolumn{2}{|c|}{ Centre offering special informative/psychological support to IMD patients during the pandemic } \\
\hline Yes & $43 / 73(58.9)$ \\
\hline No & $24 / 73(32.9)$ \\
\hline Do not know & $6 / 73(8.2)$ \\
\hline \multicolumn{2}{|c|}{ PO helping HCP in providing special support during the pandemic } \\
\hline Yes & $42 / 73(57.5)$ \\
\hline No & 21/73 (28.8) \\
\hline Do not know & $10 / 73(13.7)$ \\
\hline \multicolumn{2}{|c|}{ Active COVID-19 helpline for IMD patients in the centre } \\
\hline Yes & $38 / 73(52.1)$ \\
\hline No & $32 / 73(43.8)$ \\
\hline Do not know & $3 / 73(4.1)$ \\
\hline \multicolumn{2}{|c|}{ Patients prone to metabolic crises have the same open access to hospital as before the COVID-19 outbreak } \\
\hline Yes & $49 / 73(67.1)$ \\
\hline No & 18/73 (24.7) \\
\hline Do not know & $6 / 73(8.2)$ \\
\hline
\end{tabular}

* More than one answer possible

AOA, amino and organic acids-related disorders; PM-MD, disorder of pyruvate metabolism, Krebs cycle defects, mitochondrial oxidative phosphorylation disorders, disorders of thiamine transport and metabolism; C-FAO, carbohydrate, fatty acid oxidation and ketone bodies disorders; LSD, lysosomal storage disorders; PD, peroxisomal disorders; CDG, congenital disorders of glycosylation and disorders of intracellular trafficking; NOMS, disorders of neuromodulators and other small molecules 
cases were located in Italy (1/13), the Netherlands (1/13), and mostly in France (11/13).

In mid-April 2020, only about $10 \%$ of centers had COVID-19 cases among adult patients, for a total of 11 cases: at diagnosis, the symptoms were mild in 10 patients and severe in one case; during the infection, nine patients showed mild symptoms, and two expressed severe symptoms and were hospitalised (Table 1). Similarly to paediatric cases, the majority of adult IMD patients with COVID-19 were reported in France (6/11), followed by the UK (2/11), Belgium (2/11), and Sweden $(1 / 11)$.

No COVID-19-related deaths were reported among IMD patients (Table 1). The survey indicates an incidence of COVID-19 infection of 24/32,936 patients (72.9/100,000 equivalent for paediatric and adults, 95\% CI: 46.5-106.8/100,000) in the European IMD community.

At the beginning of the pandemic, HCPs expressed some concerns regarding the risks of severe forms of COVID-19 in IMD patients; indeed, the following IMDs are considered a major risk factor for the coronavirus disease by MetabERN experts (Table 1 and Fig. 1): amino and organic acids-related disorders (AOA) (at high risk according to $57.5 \%$ of centers); disorders of pyruvate metabolism, Krebs cycle defects, mitochondrial oxidative phosphorylation disorders, disorders of thiamine transport and metabolism (PM-MD) (56.2\%); lysosomal storage disorders (LSD) (52\%); and carbohydrate, fatty acid oxidation and ketone bodies disorders (C-FAO) (45.2\%).

\section{Management of rare metabolic patients} during the emergency

Ninety percent of MetabERN centers had to change the management of their patients due to the ongoing pandemic. In particular, changes in therapy regimen were reported by $64.4 \%$ of centers: the frequency of therapy and of rehabilitation had to be reduced in $26 \%$ and $16.4 \%$ of centers, respectively, and in $21 \%$ of centers rehabilitation was stopped (Table 1 and Fig. 2).. Where changes in the therapeutic regimes were put into place, these were unified at a regional or national level in over half of the centers (55.6\%) (Table 1). Most IMD patients (78.1\%) did not stop treatment on their own, however $14 \%$ did (Table 1).

The proportion of missed outpatient visits for IMDs was relevant, with $82 \%$ of centers reporting between 50 and $100 \%$ of missed visits (Table 1 and Fig. 3). However, almost all visits (90.4\%) were replaced by video or phone consultations (Table 1).

Despite the present emergency, the majority of HCPs (67.1\%) believe that patients with conditions prone to metabolic crises had the same access to hospitals as before the COVID-19 outbreak (Table 1).

\section{Information about COVID-19 in the rare metabolic community}

At the beginning of the emergency, good-quality, IMDspecific informative material about COVID-19 from local, national, or institutional sources was missing: many MetabERN members (45.2\%) were not aware of

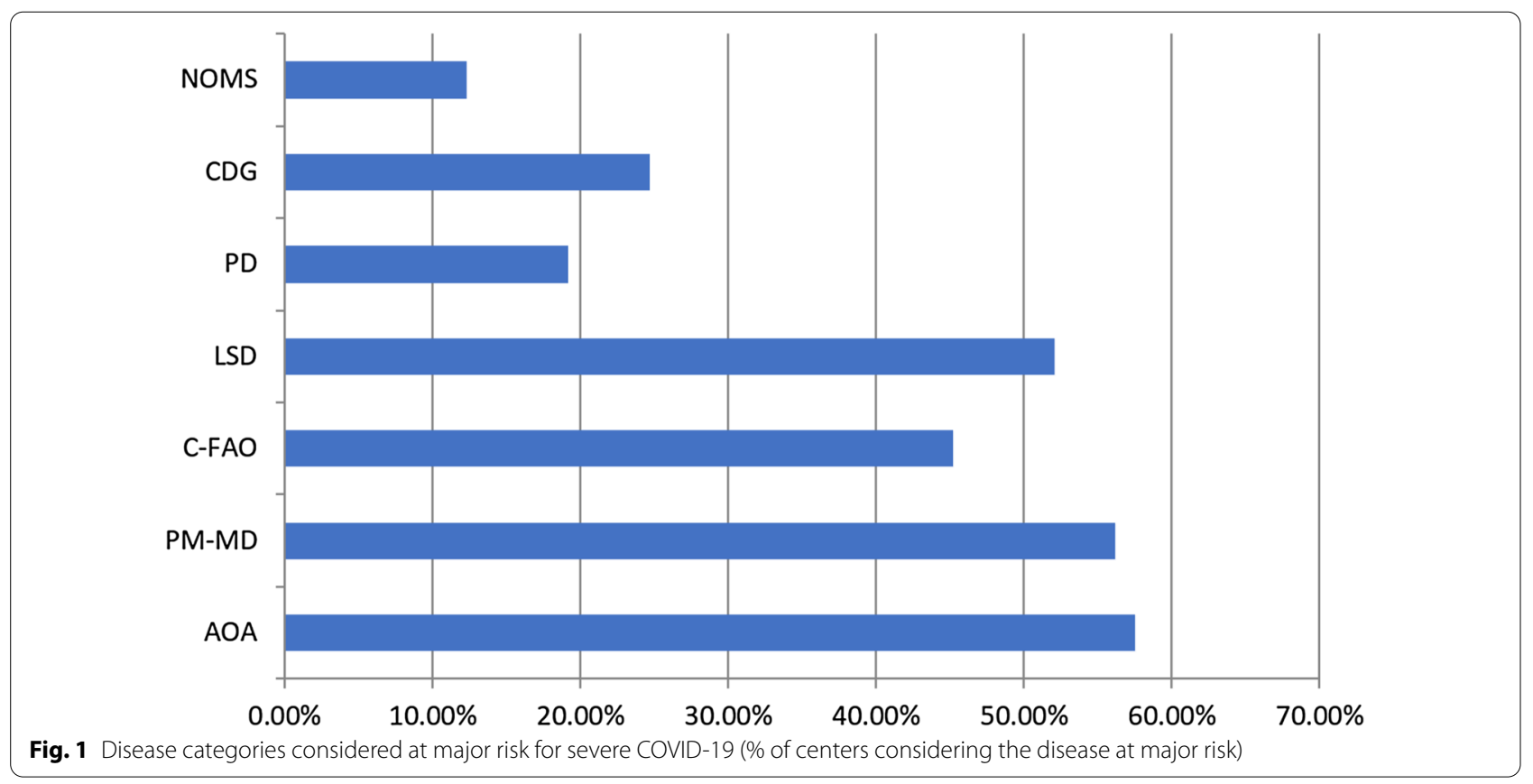




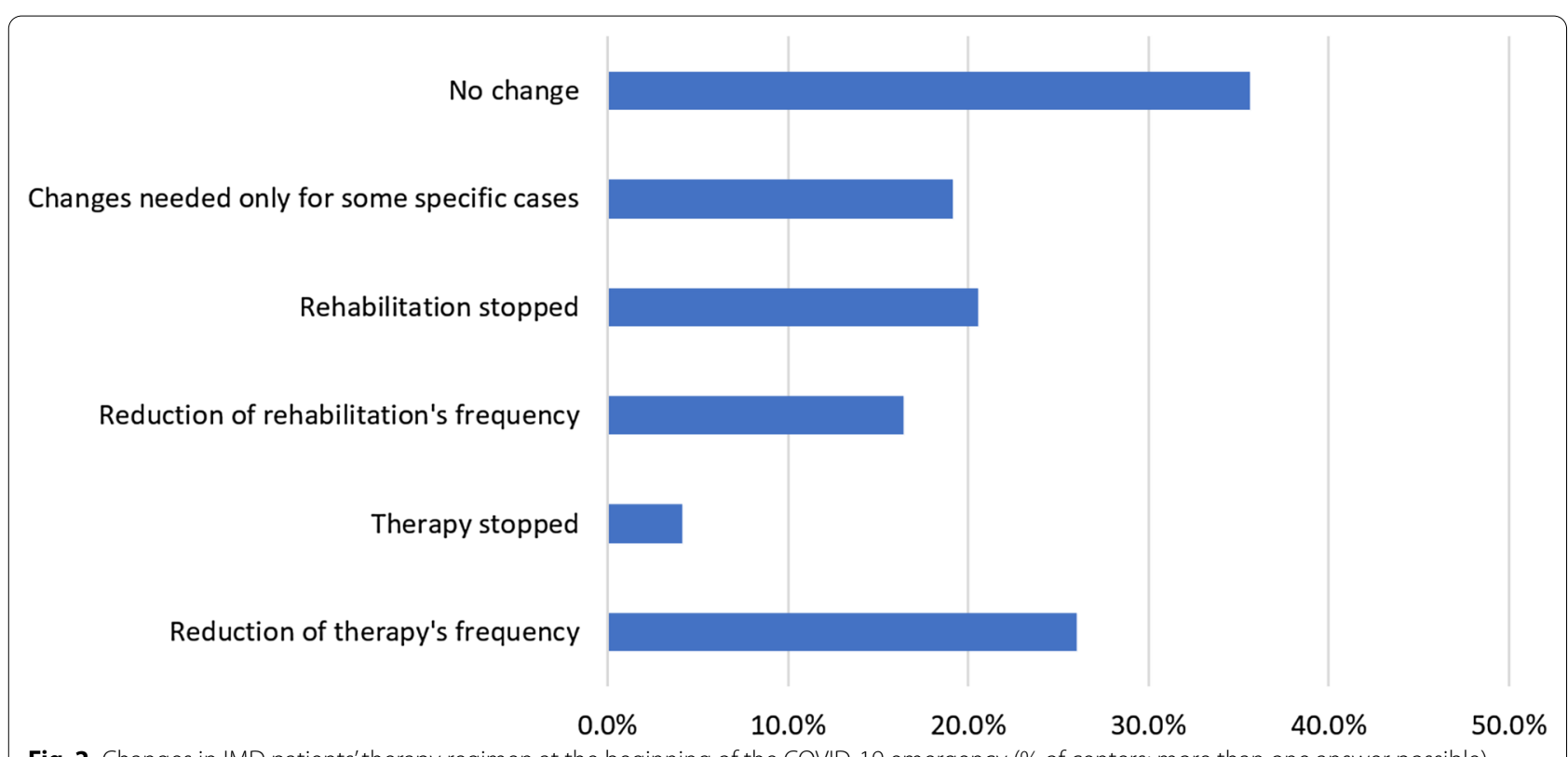

Fig. 2 Changes in IMD patients' therapy regimen at the beginning of the COVID-19 emergency (\% of centers; more than one answer possible)



this kind of information being available, and most centers (57.5\%) had not yet produced dedicated material to inform their patients (Table 1). However, in the ongoing pandemic the majority of centers $(58.9 \%)$ offered special informative and psychological support directly to their IMD patients, mostly with the help of PO (57.5\%) (Table 1). In addition, over half of the MetabERN centers (52\%) activated a COVID-19 helpline for their metabolic patients (Table 1).

\section{PO survey \\ Incidence and management of COVID-19 in rare metabolic patients}

Data relevant to the patients' perspective were collected from 39 metabolic PO from $18 \mathrm{EU}$ countries, with an $87 \%$ response rate. Similarly to HCPs, the majority of PO (87.2\%) reported no cases of COVID-19 among metabolic patients (Table 2). The PO that were aware of patients with COVID-19 in their IMD community were located in Denmark (1/5), Austria (1/5), the Netherlands (1/5), Belgium (1/5) and Italy (1/5) (no specific number of patients affected available).

Most organizations (73\%) (and/or their members) have been informed by specialized centres about COVID-19 and its risks for IMD patients, and the majority (66.7\%) also say that their patient members are in close contact with their specialized centre/HCP about the COVID-19 crisis (Table 2).

Most associations (78\%) have been contacted by some or all hospital centers in their country following their IMD members, and many organizations (48.6\%) know of HCPs that have introduced special measures or alternative care pathways to prevent SARS-CoV-2 infection in metabolic patients (Table 2): video or phone consultations, transfer to the hospital with a taxi or emergency transport, and home therapy.

Variations in visits and treatments Changes were reported in $86.5 \%$ of follow-up visits at specialized centers and in $47 \%$ of follow-up visits for clinical trials, with 


\section{Table 2 Responses collected from the PO survey}

\begin{tabular}{|c|c|}
\hline Category & n (\%) \\
\hline \multicolumn{2}{|c|}{ PO contacted by/know of IMD patient with COVID-19 } \\
\hline Yes & $5 / 39(12.8)$ \\
\hline No & $34 / 39(87.2)$ \\
\hline \multicolumn{2}{|c|}{$\mathrm{PO}$ and/or its members in close contact with the specialised centre/HCP about the COVID-19 crisis } \\
\hline Yes & $26 / 39(66.7)$ \\
\hline No & $7 / 39(18)$ \\
\hline Do not know & $6 / 39(15.3)$ \\
\hline \multicolumn{2}{|c|}{ PO informed by specialist centre about COVID-19 and its risk for IMD patients } \\
\hline Yes & 27/37 (73) \\
\hline No & $10 / 37(27)$ \\
\hline \multicolumn{2}{|c|}{ Centers following IMD patients in the country that are in contact with the PO } \\
\hline All of them & $10 / 36(27.8)$ \\
\hline Most of them ( $\geq 50 \%)$ & $8 / 36(22.2)$ \\
\hline A few $(<50 \%)$ & $10 / 36(27.8)$ \\
\hline None & $8 / 36(22.2)$ \\
\hline \multicolumn{2}{|c|}{ Centers have put into place measures or alternative pathways to prevent COVID-19 in IMD patients } \\
\hline Yes, all of them & 10/37 (27) \\
\hline Yes, some of them & $8 / 37(21.6)$ \\
\hline Almost none & 2/37 (5.4) \\
\hline None & $4 / 37(10.8)$ \\
\hline Do not know & $13 / 37(35.1)$ \\
\hline \multicolumn{2}{|l|}{ PO's members experiencing changes } \\
\hline \multicolumn{2}{|l|}{ In follow up visits at the specialised centre } \\
\hline Yes & $32 / 37(86.5)$ \\
\hline No & $1 / 37(2.7)$ \\
\hline Do not know & $4 / 37(10.8)$ \\
\hline \multicolumn{2}{|c|}{ In follow up visits for clinical trials at the specialised centre } \\
\hline Yes & $14 / 30(46.7)$ \\
\hline No & $3 / 30(10)$ \\
\hline Do not know & $13 / 30(43.3)$ \\
\hline \multicolumn{2}{|l|}{ Type of changes* } \\
\hline Cancellations of outpatient stays & $17 / 31(54.8)$ \\
\hline Cancellations of inpatient stays & 10/31 (32.3) \\
\hline Postponing of appointments & 28/31 (90.3) \\
\hline \multicolumn{2}{|c|}{ Changes in IMD treatment due to COVID-19 pandemic } \\
\hline Yes & 20/39 (51.3) \\
\hline No & $19 / 39(48.7)$ \\
\hline \multicolumn{2}{|l|}{ Type of changes* } \\
\hline Discontinuation & $13 / 20(65)$ \\
\hline Prolonged time between treatments & $7 / 20(35)$ \\
\hline Switch to home therapy & $10 / 20(50)$ \\
\hline Problems in getting the medication & $5 / 20(25)$ \\
\hline \multicolumn{2}{|c|}{ Therapies (physio, speech, or enzyme replacement therapy, etc.) continued } \\
\hline Yes & 10/38 (26.3) \\
\hline No & $16 / 38(42.1)$ \\
\hline Do not know & $12 / 38(31.6)$ \\
\hline \multicolumn{2}{|c|}{ Psychological support available for patients scared about COVID-19 } \\
\hline Yes & 28/39 (71.8) \\
\hline No & $11 / 39(28.2)$ \\
\hline
\end{tabular}


Table 2 (continued)

\begin{tabular}{ll}
\hline Category & $\mathbf{n}(\%)$ \\
\hline Specialist/entity providing psychological support* & 8/32(25) \\
Physician & $11 / 32(34.4)$ \\
Psychologist & $7 / 32(21.9)$ \\
Social worker & $21 / 32(65.6)$ \\
PO & $13 / 32(40.6)$ \\
Other & $25 / 31(80.7)$ \\
Method/technology used to provide psychological support* & $20 / 31(64.5)$ \\
Phone & $15 / 31(48.4)$ \\
Mail/Email & $9 / 31(29)$ \\
Video & \\
Other & \\
\hline
\end{tabular}

* More than one answer possible

respectively $55 \%$ and $32 \%$ of cancelled outpatients and inpatient stays, and $90 \%$ of postponed appointments (Table 2 and Fig. 4).

Over half of PO (51.3\%) reported changes also in IMD treatments due to COVID-19, in particular: discontinuation $(65 \%)$, change to home treatment $(50 \%)$, prolonged time between treatments (35\%), and problems in getting the medication (25\%) (Table 2 and Fig. 5). Only 26\% of organizations reported no interruption of therapy (e.g. physiotherapy, speech therapy, enzyme replacement therapy, etc.), while $42 \%$ said these were stopped (Table 2).

Support and concerns about COVID-19 in rare metabolic patients Seventy-two percent of PO confirmed that psychological support was provided to patients worried about the COVID-19 outbreak, mostly through the PO themselves (65.6\%) via phone, video, or email (Table 2).

According to PO, besides the possibility of being infected with COVID-19, most patients were also worried about not having access to therapy or to the same quality of care as before. In addition they were concerned about additional pressure put on family caregivers, anxiety and mental health issues related to quarantine and care limitations (Table 3 and Fig. 6).

\section{Discussion}

The coronavirus pandemic has taken healthcare system all over the world by surprise: totally unprepared, HCPs had to rapidly change the way they organize and deliver medical care, not only to assist COVID-19 cases, but for all patients. As such, the pandemic is having a significant impact on patients affected by chronic and multiorgan disorders such as IMDs. In the general population, the most severe and fatal cases of COVID-19 are observed in patients $60+$ years of age and in those with underlying health conditions (diabetes, hypertension, cardiac disorders, chronic lung disease), while children are less frequently infected and present milder symptoms than adults [4]. In the rare metabolic community, where all patients, including pediatric ones, experience multiorgan impairments caused by their chronic condition, the risk of severe forms of COVID-19 may be high. Indeed, at the early stage of the pandemic, MetabERN experts were concerned regarding the potential effect of the infection on their patients, as those with an IMD have a genetic condition that predisposes them to metabolic destabilization and gradual worsening of the clinical course, with progressive neurological deterioration and multi-organ dysfunction; for these reasons, constant controls, diet corrections, and therapy are critical for the wellbeing and, in some cases, survival of IMD patients. Therefore, recommendations to decrease the infection rate were expressed by the network.

In the present situation, rare metabolic patients need to be protected not only from the risk of coronavirus infection, but also from the possible degeneration of their disease. To this end, a network of HCPs in close contact with IMD patients and their caregivers is essential to provide patients with the necessary support and care. Indeed, our two surveys revealed a close interaction of HCPs with their patients and the PO during this time of crisis. At the same time, the HCPs survey showed that at the beginning of the coronavirus emergency (March-April 2020) the incidence of COVID-19 among IMD patients was lower than that of the general European population (EU/ EEA and UK) reported from 1st January to 7th April 2020 (72.9/100,000 vs. 117/100,000) [9]. Both aspects suggest the existence of a strong IMD community, supported by a solid network of HCPs and PO that were able to act quickly and effectively to protect metabolic patients from SARS-CoV-2 infection-at least to a certain extent. In an unprecedent situation with a lot of unknowns, during 


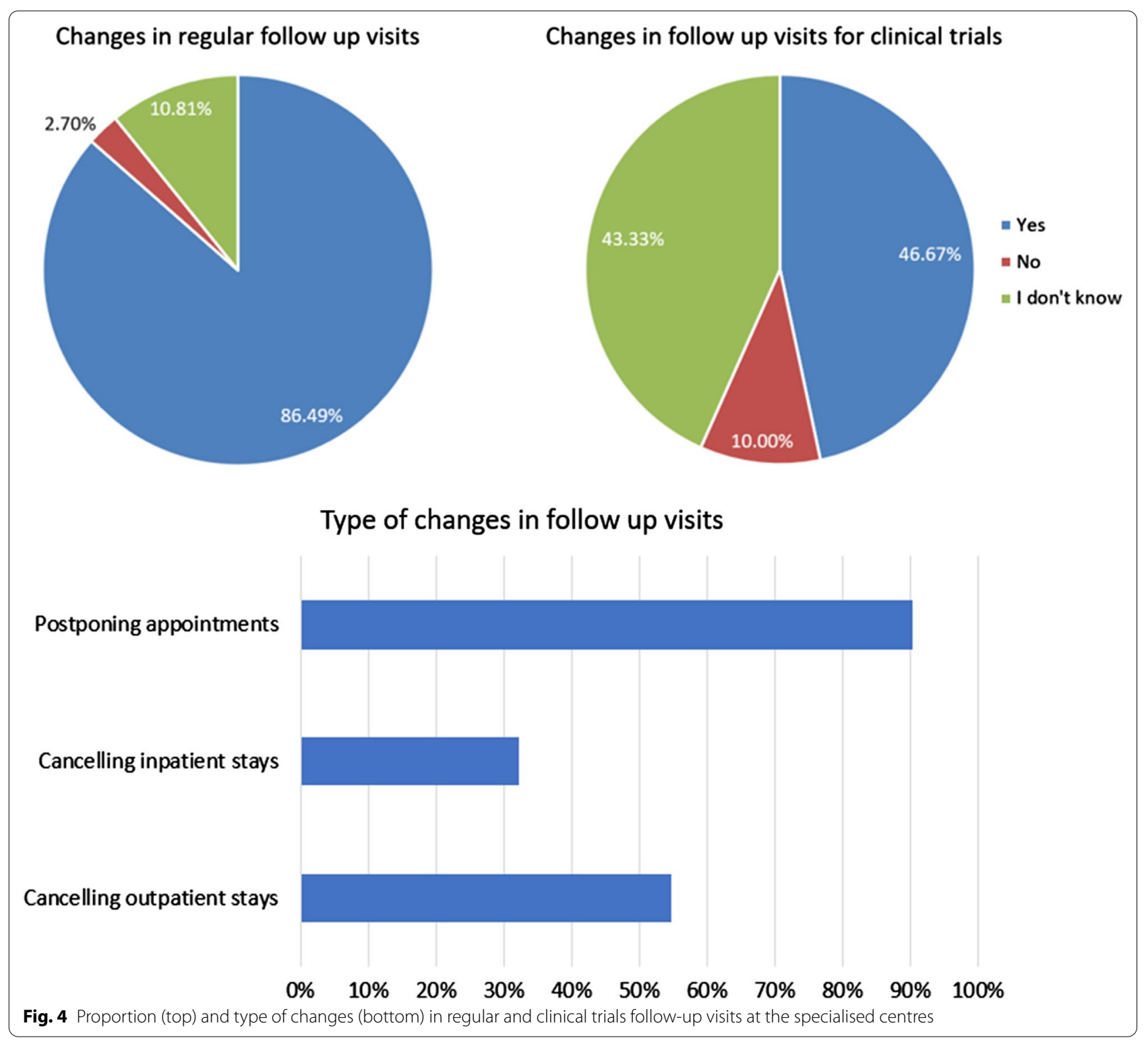

which specific information from health institutions and scientific literature about COVID-19 in metabolic diseases was not available or limited, HCPs contacted the patients directly to provide the necessary support and information. As the situation rapidly evolved, informative material on coronavirus and IMDs became available from local and international institutions and organizations; MetabERN collected the links to these resources on its website and also published recommendations, Q\&A and infographics about COVID-19 in rare metabolic patients [10]. In general, IMD patients receive detailed information from specialists to avoid any metabolic decompensation due to other diseases or infections. For COVID-19, it was not necessary to provide additional indications besides the general information given at the local or national level. Importantly, to help the entire metabolic community, MetabERN has developed some recommendations to support all IMD patients and caregivers during the COVID-19 emergency. These recommendations stress the importance of sustaining efforts to prevent, diagnose and treat IMDs, assuring the continuation of the quality of care provided to patients and recommending, whenever possible, to direct patients to home therapy and to invite them to go to the hospital for a visit only if really needed. In particular, the use of videoconferences as a replacement for many of the face-to-face meetings is encouraged. 


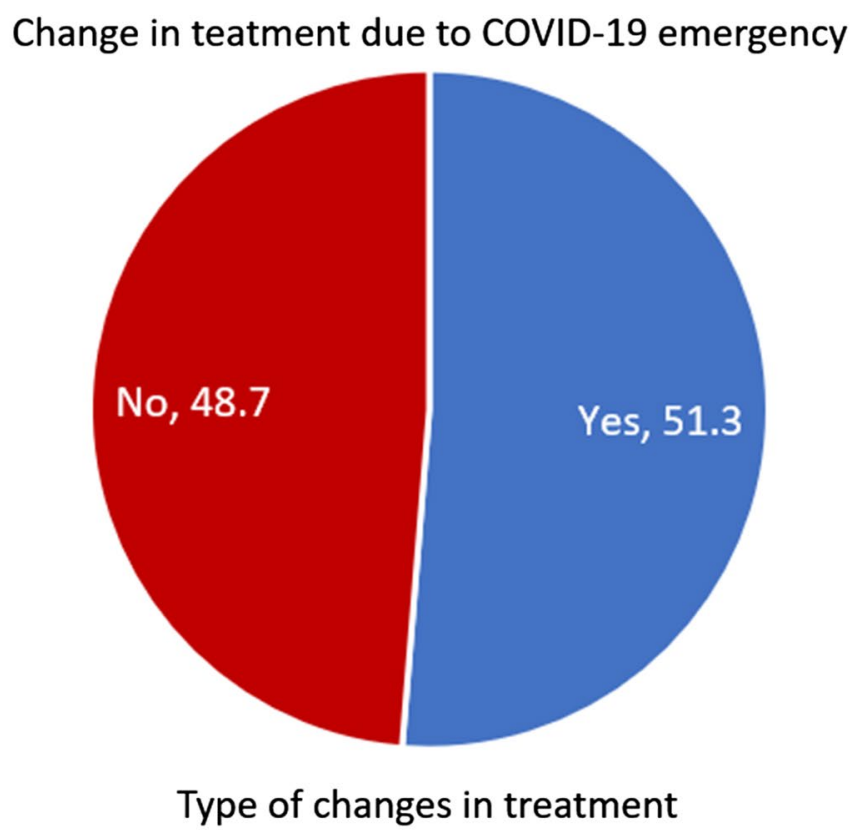

Problems in getting the medication

Switch to home therapy

Prolonged time between treatments

Discontinuation



$0 \%$

$10 \% \quad 20 \%$

$30 \%$

$40 \%$

$50 \%$

$60 \%$

$70 \%$

Fig. 5 Proportion (top) and type of changes (bottom) in IMD treatment due to the COVID-19 emergency

Table 3 Description and levels of concern of the IMD patient community

\begin{tabular}{|c|c|c|c|c|c|}
\hline \multirow[t]{2}{*}{ Description of concern } & \multicolumn{5}{|c|}{ Level of concern } \\
\hline & $\begin{array}{l}\text { Not } \\
\text { worried } \\
\text { at all }\end{array}$ & $\begin{array}{l}\text { Somewhat } \\
\text { worried }\end{array}$ & $\begin{array}{l}\text { Neither } \\
\text { worried } \\
\text { nor unworried }\end{array}$ & Worried & Extremely worried \\
\hline Having the therapy suspended & $\begin{array}{l}1 / 3 \\
(2.8)\end{array}$ & $\begin{array}{l}8 / 36 \\
(22.2)\end{array}$ & $\begin{array}{l}3 / 36 \\
(8.3)\end{array}$ & $\begin{array}{l}14 / 36 \\
(38.9)\end{array}$ & $\begin{array}{l}10 / 36 \\
(27.8)\end{array}$ \\
\hline Not having access to the same standard quality of care as before & $\begin{array}{l}2 / 36 \\
(5.6)\end{array}$ & $\begin{array}{l}2 / 36 \\
(5.6)\end{array}$ & $\begin{array}{l}6 / 36 \\
(16.7)\end{array}$ & $\begin{array}{l}16 / 36 \\
(44.4)\end{array}$ & $\begin{array}{l}10 / 36 \\
(27.8)\end{array}$ \\
\hline $\begin{array}{l}\text { Anxiety and mental health issues related with the quarantine and current } \\
\text { medical care limitations }\end{array}$ & $\begin{array}{l}0 / 35 \\
(0)\end{array}$ & $\begin{array}{l}6 / 35 \\
(17.1)\end{array}$ & $\begin{array}{l}6 / 35 \\
(17.1)\end{array}$ & $\begin{array}{l}18 / 35 \\
(51.4)\end{array}$ & $\begin{array}{l}5 / 35 \\
(14.3)\end{array}$ \\
\hline Possibility of getting COVID-19 & $\begin{array}{l}0 / 35 \\
(0)\end{array}$ & $\begin{array}{l}4 / 35 \\
(11.4)\end{array}$ & $\begin{array}{l}2 / 35 \\
(5.7)\end{array}$ & $\begin{array}{l}12 / 35 \\
(34.3)\end{array}$ & $\begin{array}{l}17 / 355 \\
(48.6)\end{array}$ \\
\hline Additional strain being put on family caregivers & $\begin{array}{l}0 / 34 \\
(0)\end{array}$ & $\begin{array}{l}5 / 34 \\
(14.7)\end{array}$ & $\begin{array}{l}3 / 34 \\
(8.8)\end{array}$ & $\begin{array}{l}19 / 34 \\
(55.9)\end{array}$ & $\begin{array}{l}7 / 34 \\
(20.6)\end{array}$ \\
\hline $\begin{array}{l}\text { Patients/caregivers not having access to reliable and comprehensible } \\
\text { information }\end{array}$ & $\begin{array}{l}5 / 36 \\
(13.9)\end{array}$ & $\begin{array}{l}6 / 36 \\
(16.7)\end{array}$ & $\begin{array}{l}10 / 36 \\
(27.8)\end{array}$ & $\begin{array}{l}9 / 36 \\
(25)\end{array}$ & $\begin{array}{l}6 / 36 \\
(16.7)\end{array}$ \\
\hline
\end{tabular}


Patients/caregivers not having access to reliable and comprehensible information

Additional strain being put on family caregivers

Possibility of getting COVID-19

Anxiety and mental health issues related with the quarantine and current medical care limitations

Not having access to the same standard quality of care as before

Having the therapy suspended

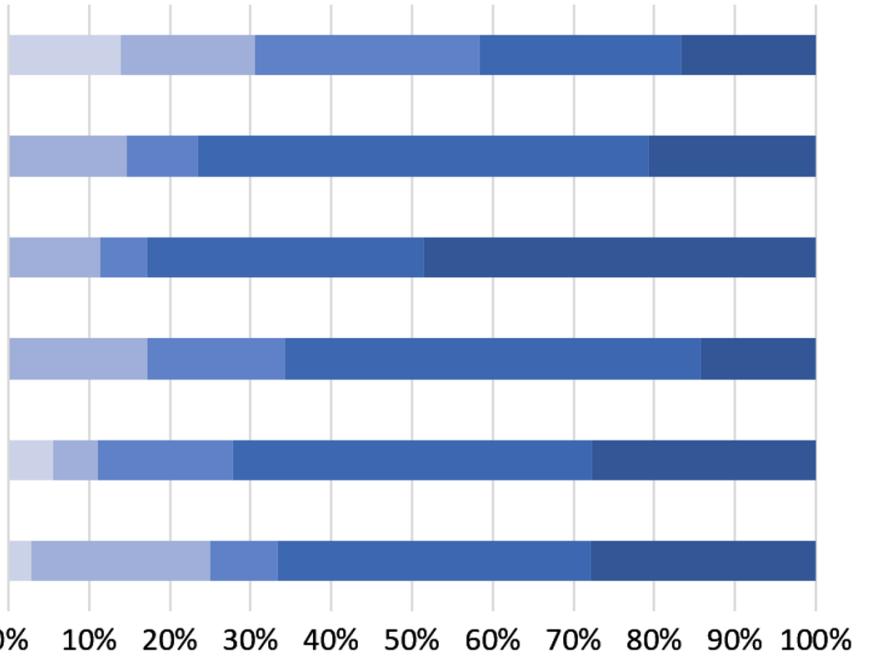

Not worried at all Somewhat worried $\square$ Neither worried nor unworried $\square$ Worried $\square$ Extremely worried
At the very beginning HCPs did not know what kind of disease COVID-19 was, so the information was based on avoiding decompensation. Now that HCPs know what disease it is, they give more precise recommendations on how to manage fever to avoid metabolic decompensation.

According to the data collected through our HCPs survey, COVID-19 cases in IMD patients were registered in six EU countries: Belgium, Italy, the Netherlands, Sweden, the UK, and especially France, where most of the cases were reported (17 out of 24). This may suggest that the IMD community in France was more heavily hit by the pandemic than the rest of the EU; however, these results may be linked to a better identification of COVID19 cases in France compared to other countries thanks to a more rigorous reporting to HCPs by patients, a better reporting of cases to MetabERN by French specialists, or an actual higher number of cases. Unfortunately, our data are too small to reach statistical significance, so they cannot be used to make comparisons or draw any conclusions on the national incidence of COVID-19 among IMD patients. In addition, our data may be incomplete and reflect only a fraction of the national situation reported by MetabERN centres; plus, in some European countries testing for COVID-19 might have been limited or unavailable at the time of our survey.

HCPs data on COVID-19 cases differ from that collected from the PO, which reported COVID-19 patients within the IMD community in five countries: Denmark, Austria, the Netherlands, Belgium and Italy-notably, not France. This result suggests that, while communication between HCPs and PO on the patients' management in general and in emergency situations is rapid and up-to-date, communication on specific clinical cases is lacking. Also, it is difficult to define the interaction between HCPs and PO, and between PO and patients: some PO interact often with HCPs and their patients, others do not; similarly, not all patients are in regular contact with their association.

As a mean to protect IMD patients from COVID-19, visits were mostly cancelled or postponed: more than half of HCPs reported $75-100 \%$ of missed outpatient visits, confirming data gathered from the PO, which reported changes in $87 \%$ of follow-up visits (and in $47 \%$ of clinical trials visits), with $90 \%$ of postponed appointments and $55 \%$ of cancelled outpatients stays. These results are in line with other surveys performed on the broader rare disease community by EURORDIS, NORD and Rare Disease Ireland. According to these studies, $80 \%$ of patients had their rehabilitation postponed or cancelled [5], and $50-70 \%$ had their appointments with the general practitioner or specialist cancelled $[5,6,8]$. As SARS-CoV-2 reached Europe and governments and healthcare systems struggled to contain the spread of the virus, cancelling or postponing face-to-face visits was the most obvious, immediate and critical action to put into place to limit infections and protect all patients, including those with IMDs.

Similarly, most treatments were reduced or suspended: a quarter of HCPs reduced the frequency of therapy, stopping it in only $4 \%$ of cases, while rehabilitation was reduced or stopped in $16 \%$ and $21 \%$ of cases, respectively. Indeed, according to the PO, over half of IMD patients experienced a change in treatment: $65 \%$ faced discontinuation; $50 \%$ switched to home therapy; 
$35 \%$ prolonged the time between treatments; and 25\% had problems in getting the medication. The results on the discontinuation of care are in line with recent data about the effects of the pandemic in Italy, where $52 \%$ of rare disease patients suspended hospital therapy [7] and 49\% of LSD patients receiving enzyme replacement therapy in hospital experienced disruption [11]. Considering the huge impact of COVID-19 on hospitals' organization and workload, it is not surprising that in many cases treatments for rare disease patients had to be halted or rearranged to limit the infection and to divert resources towards COVID-19 patients. However, our results are somehow different from those of the EURORDIS survey: while this reported that $60 \%$ of rare disease patients had no access to therapies such as infusions, chemotherapy, and hormonal treatment at home or at the hospital [5], we found that about half of IMD patients were able to switch to home therapy. This difference may be due to the fact that home treatment is an option for IMDs, but not for other rare diseases. Also, national healthcare systems in EU countries were re-organised differently during the emergency, and this may have impacted the availability of personnel, medical equipment and drugs for rare diseases.

Our data also show that about a quarter of patients are encountering difficulties in accessing medicines. This result is similar to what was reported in Ireland [8] and Italy [7] for rare diseases in general, but it differs from the situation of LSD therapies in Italy, where there were no problems in medicine supply, even in the most affected regions [11]. These differences are most likely a reflection of the different national healthcare settings across Europe and of the different drugs used by rare diseases patients. Still, the situation is worrisome and calls for an immediate and coordinate action by national health systems.

Importantly, our surveys show that in most cases (78\%) rare metabolic patients did not stop treatment by their own decision: a higher percentage than that reported in the Italian survey on rare disease patients (44\%) [7]. This aspect is a further evidence of the strong collaboration that exists between HCPs and IMD patients.

The immediate consequences of visits and treatments disruption for the patients were mitigated by the widespread use of telemedicine among HCPs, as $90 \%$ of centres were able to replace face-to-face visits with video or phone calls during the emergency. This percentage is much higher than that observed in the general rare disease community in Europe and in the United States, where $30-60 \%$ of patients used telemedicine or other forms of remote consultation [5, 6, 8]. With telemedicine becoming more and more relevant for the future of healthcare, reducing physical and geographical barriers, this result demonstrates that the IMD community is ready to adopt current and novel digital tools to ensure constant and adequate care to all metabolic patients.

The main fears of IMD patients include getting COVID-19, not having access to the same quality of care as before the pandemic, and having the therapy suspended. In normal circumstances, rare metabolic patients face constant challenges to maintain their wellbeing and avoid a degeneration of their condition, so these results are not surprising given the significant changes imposed by the pandemic on all aspects of daily life. Indeed, other surveys found that the majority of rare disease patients are worried about the impact of COVID19 on their disease-being it direct infection and/or indirect interference with the management and treatment of the disease $[5,6,8]$. The concerns of IMD patients are somewhat in line with those expressed by cancer and diabetic patients in Europe: recent surveys have showed that people with cancer in the Netherlands and the UK are mostly concerned about the impact of the pandemic on their treatment or follow-up [12] and are worried about catching COVID-19 and becoming seriously ill from this disease [13]; similarly, the main concern of patients with type 1 diabetes in Denmark is to be overly affected due to diabetes if infected with SARS-CoV-2 [14].

The model of care established within the rare metabolic community is based on a close collaboration between HCPs, patients and local PO; during the unprecedent crisis caused by SARS-CoV-2, the results of our surveys suggest that this model was successful in protecting IMD patients from the infection. More time is needed to determine the incidence of COVID-19 in rare metabolic patients and to fully assess the mid- and long-term impact of the pandemic on their wellbeing. As a first step in addressing this issue, we have already developed a second survey for HCPs to better understand the number of COVID-19 cases in IMD patients, and the clinical manifestations and consequences of the infection in these patients across Europe. As the pandemic progresses, various measures can be put into place to mitigate its impact on IMD patients and alleviate the shortcomings identified in our surveys: each hospital should setup appropriate and well-identified COVID-19-free paths and areas to allow a safe use of healthcare services by patients not infected by SARS-CoV-2; home therapy should be expanded as much as possible to ensure treatment to all patients; telemedicine tools should be implemented widely to provide clinical and also psychological support to patients and their caregivers; and additional daily assistance (through nurses or social assistants) should be offered to those with neurological and/or highly debilitating IMDs. 
Our study has several limitations: first, as mentioned above, the data collected is too small to make significant statistical analyses focused on the single country or region; therefore, while we were able to identify some trends at a European level, we cannot make any reliable considerations at a national level. Furthermore, the data were generated inside the MetabERN, so we cannot expand our conclusions outside our network of HCPs and patients. Secondly, we were able to collect only qualitative data on the risk of severe COVID-19 in IMD patients: HCPs gave their opinion on whether having an IMD increases this risk, but no quantitative data is currently available to support these opinions. To gather the necessary quantitative data we have already distributed among MetabERN members a second survey on COVID-19, which focuses on the epidemiology and the clinical consequences of SARS-CoV-2 infection in rare metabolic patients. Thirdly, while the PO survey was extremely useful to understand the impact of the coronavirus pandemic from the patients' perspective, the data collected is limited to the amount of information available to the $\mathrm{PO}$, which may be incomplete and not up to date compared to what HCPs know.

The continuing disruption of the proper follow-up of IMD patients might compromise their clinical condition and aggravate the course of the metabolic disease, from a medical, psychological, and also social point of view. Therefore, HCPs and PO need to keep working together to monitor the health status of their patients and provide the necessary care using the available telemedicine tools; at the same time, long-term adjustments must be put into place by national health systems to restore continuous therapies and treatment for IMD patients.

\section{Conclusion}

We provide the first and most comprehensive evidence of the impact of COVID-19 on the rare metabolic community. During the initial phase of the coronavirus pandemic, the highly medically supervised population of IMD patients was relatively protected from the infection. This was made possible also by the widespread use of telemedicine in the metabolic community. Indeed, digital tools have, to some extent, the capacity to ensure the continuity of care, not only in critical situations like a pandemic, but also for standard healthcare. More time is needed to determine the actual consequences of the COVID-19 emergency on IMD patients.

\section{Supplementary information}

Supplementary information accompanies this paper at https://doi. org/10.1186/s13023-020-01619-x.

Additional file 1. Original survey sent to HCPs. List of questions and possible answers included in the HCPs survey.

Additional file 2. Original survey sent to PO. List of questions and possible answers included in the PO survey.

\section{Abbreviations}

WHO: World Health Organization; IMD: Inherited metabolic diseases; PO: Patients organizations; HCP: Healthcare provider; ERN: European Reference Network; MetabERN: European Reference Network for Hereditary Metabolic Diseases.

\section{Acknowledgements}

We thank Francesca Valent for the revision of our statistical analysis and the HCP representatives of the MetabERN collaboration group that replied to our survey: Florian Lagler, Universitätsklinikum der Paracelsus Medizinischen Privatuniversität, Salzburg, Austria; Greber-Platzer Susanne, Klinische Abteilung für Pädiatrische Pulmologie, Allergologie und Endokrinologie, Vienna, Austria; Dorothea Möslinger, Universitätsklinik für Kinder- und Jugendheilkunde Allgemeines Krankenhaus der Stadt Wien, Austria; François-Guillaume Debray, CHU Liège, Belgium; Marie-Cécile Nassogne, Cliniques universitaires St Luc (Bruxelles)-Université catholique de Louvain, Belgium; Corinne de Laet, Metabolic Center ULB-VUB, Belgium; François Eyskens, University Hospital of Antwerp UZA, Belgium; Peter Witters, UZ Leuven, Belgium; Dominique Roland, nstitut de Pathologie et de Génétique, Gosselies, Belgium; Patrick Verloo, Ghent University Hospital, Belgium; Tessa Wassenberg, Vrije Universititeit Brussel, Belgium; Teodora Chamova, Alexandrovska University Hospital, Sofia, Bulgaria; Tomas Honzik, Charles University and General University Hospital, Prague, Czech Republic; Danijela Petković Ramadža, University Hospital Center Zagreb, Croatia; Allan Meldgaard Lund, Copenhagen University Hospital, Denmark; Dominique Germain, Assistance Publique-Hôpitaux de Paris lle de France Ouest, France; Nadia Belmatoug, Assistance Publique-Hôpitaux de Paris Nord Val de Seine Beaujon, France; Philippe Labrune, Assistance PubliqueHôpitaux de Paris Antoine-Béclère, France; Pascale de Lonlay, Assistance Publique-Hôpitaux de Paris Necker-Enfants Malades, France; Dries Dobbelaere, University Hospital of Lille, France; Aline Cano, Assistance Publique-Hôpitaux de Marseille, France; Anihb Martin Das, Hannover Medical School, Germany; Frank Rutsch, Muenster University Hospital, Germany; Klaus Mohnike, Ottovon-Guericke-University, Germany; Stefan Kölker, Universitätsklinikum Heidelberg, Germany; Julia Hennermann, University Medical Center Mainz, Germany; Nicole Muschol, Universitätsklinikum Hamburg-Eppendorf, Germany; Sarah Grünert, Universitätsklinikum Freiburg, Germany; Katalin Szakszon, University of Debrecen, Hungary; Serena Gasperini, ASST Monza San Gerardo Hospital, Italy; Giancarlo Parenti, Azienda Ospedaliera Universitaria "Federico II" Napoli, Italy; Alessandro Simonati, Azienda Ospedaliera Universitaria Integrata di Verona, Italy; Maja di Rocco, Giannina Gaslini Institute, Italy; Graziella Cefalo, San Paolo Hospital, ASST Santi Paolo e Carlo, Italy; Giovanni Ceccarini, Azienda Ospedaliero-Universitaria Pisana, Italy; Salvatore Grosso, Azienda Ospedaliera Universitaria Senese, Italy; Laura Rubert, Fondazione IRCCS Policlinico San Matteo, Italy; Andrea Bordugo, Azienda Ospedaliera Universitaria Integrata di Verona - Ospedale Borgo Roma, Italy; Pietro Strisciuglio, University of Naples, Italy; Flavia Tubilli, Ospedale Pediatrico Meyer, Florence, Italy; Ferruccio Santini, Azienda Ospedaliero-Universitaria Pisana, Italy; Madara Masinska, Bērnu Klīniskā Universitātes Slimnīca, Riga, Latvia; Nicola Dingli, Mater Dei Hospital, Malta; Ans van der Ploeg, Erasmus MC, University Medical Center, Rotterdam, Netherlands; Maria-Estela Rubio-Gozalbo, Maastricht University Medical Center, Netherlands; Francian van Spronsen, University of Groningen Medical Center, Netherlands; Hidde Huidekoper, Erasmus MC, University Medical Center, Rotterdam, Netherlands; Marc Engelen, Emma Kinderziekenhuis Amsterdam UMC, Netherlands; Terry Derks, Universitair Medisch Centrum 
Groningen, Netherlands; Trine Tangeraas, Oslo University Hospital, Norway; Sverre Sandberg, Helse Bergen HF, Haukeland University Hospital, Norway; Aasne Aarsand, Haukeland University Hospital, Bergen, Norway; Beata KiećWilk, Krakow University Hospital, Poland; Esmeralda Martins, Centro Hospitalar do Porto, Portugal; Esmeralda-Maria Ferreira Rodrigues Silva, Centro Hospitalar do Sao Joao, Portugal; Luísa-Maria de Abreu Freire Diogo Matos, Centro Hospitalar e Universitário de Coimbra, Portugal; Olga Azevedo, Hospital Senhora da Oliveira - Guimarães, Portugal; Maria Carmo Macàrio, Hospitais da Universidade de Coimbra, Portugal; Elisa Leão Teles, Centro Hospitalar de S. João, Porto, Portugal; Simona Tarnokova, Detska fakultna nemocnica s poliklinikou Bratislava, Slovakia; Mojca-Zerjav Tansek, University Medical Centre Ljubljana, Slovenia; Urh Groselj, University Medical Centre Ljubljana, Slovenia; Maria-Luz Couce-Pico, Complejo Hospitalario Universitario de Santiago de Compostela, Spain; Angeles Garcia Cazorla, Hospital Sant Joan de Déu, Spain; Mireia del Toro-Riera, Hospital Universitario Vall d'Hebron, Spain; Montserrat Morales Conejo, Hospital Universitario 12 de Octubre, Madrid, Spain; Paula Sànchez Pintos, Hospital Clínico Universitario de Santiago, Spain; Svetlana Lajic, Karolinska University Hospital, Sweden; Niklas Darin, Sahlgrenska University Hospital and Sahlgrenska Academy at the University of Gothenburg, Sweden; Charlotte Dawson, University Hospital Birmingham, United Kingdom; Elizabeth Morris, Addenbrooke's Hospital, Cambridge, United Kingdom. We also thank the PO that replied to our survey: Gesellschaft für Mukopolysaccharidosen und ähnlich Erkrankungen, Austria; Fett-SOS e.V. (Selbsthilfegruppe für angeborene FettSäurenOxidationsStörungen), Austria and Germany; BOKS vzw, Belgium; ASPIDA ZOIS, Cyprus Association of Inherited Metabolic Diseases, Cyprus; Rare Diseses Croatia, Croatia; META z.S., Czech Republic; Ketotic Hypoglycemia International, Denmark; Gaucher Foreningen i Danmark, Denmark; Finnish Fabry Association, Finland; MSD Action Foundation, France; VML, France; Association Francophone des Glycogénoses (AFG), France Deutschsprachige Selbsthilfegruppe für Alkaptonurie e.V., Germany; Deutsche Interessengemeinschaft für Phenylketonurie (DIG PKU), Germany; SLO Deutschland, Germany; Eigeninitiative Smith Lemli Opitz Syndrom, Germany; Pompe Deutschland, Germany; KRIKOS ZOIS, Greece; Greek Lysosomal Association, Greece; Associazione Italiana Malati di Alcaptonuria (aimAKU), Italy; Associazione Italiana Gaucher, Italy; LND Famiglie Italiane, Italy; MItocon, Italy; Associazione Italiana Gaucher ONLUS, Italy; ALAN asbl Maladies Rares Luxembourg, Luxembourg; Volwassenen, Kinderen en Stofwisselingsziekten (VKS), Netherlands; FSIGN, Netherlands; Spanish MPS Society, Spain; Asociaciòn Española Sindrome CDG (AESCDG), Spain; JMR Foundation for persons with SMith Lemli Opitz syndrome, Sweden; Swedish MPS society, Sweden; Portuguese Association for CDG, Portugal; Portuguese Association for CDG, Portugal; Gauchers Association, United Kingdom; Niemann-Pick UK, United Kingdom; CDG UK, United Kingdom.

\section{Authors' contributions}

$A F, C B, R F, S B$ and $S S$ revised and distributed the PO survey. CL prepared the surveys and revised the manuscript. CMB prepared the surveys, analysed the data, and revised the manuscript. CVL revised the manuscript. JMH analysed the data and revised the manuscript. LP analysed the data and wrote the manuscript. MS and CD-V conceived the study, prepared the surveys and revised the manuscript. All authors read and approved the final manuscript.

\section{Funding}

This work was generated within the European Reference Network for Rare Hereditary Metabolic Disorders (MetabERN), co-funded by the European Union within the framework of the Third Health Programme ERN-2016 Framework Partnership Agreement 2017-2021, Project ID No. 739543.

\section{Availability of data and materials}

The datasets used and/or analysed during the current study are available from the corresponding author on reasonable request.

\section{Ethics approval and consent to participate}

All surveys' participants gave their consent for data collection and publication.

\section{Competing interests}

The authors declare that they have no competing interests.

\section{Author details}

${ }^{1}$ Center for Rare Diseases Giessen (ZSEGI), University Hospital Giessen, Giessen, Germany. ${ }^{2}$ Division of Metabolism, Bambino Gesù Children's Hospital, IRCCS, Rome, Italy. ${ }^{3}$ MetabERN, Regional Coordinating Center for Rare Diseases, Udine University Hospital, Udine, Italy. ${ }^{4}$ Krabbe UK, London, UK. ${ }^{5}$ MSD Action Foundation, Dublin, Ireland. ${ }^{6}$ Portuguese Association for CDG and Other Rare Metabolic Diseases (APCDG-DMR), Almada, Portugal. ${ }^{7}$ Italian Association of Patients With Alkaptonuria (aimAKU), Siena, Italy.

Received: 24 August 2020 Accepted: 16 November 2020

Published online: 03 December 2020

\section{References}

1. World Health Organization. Coronavirus disease 2019 (COVID-19) Situation Report-51. 2020; 11/03/2020.

2. World Health Organization. WHO Coronavirus Disease (COVID-19) Dashboard. 2020. https://covid19.who.int/. Accessed 20 Oct 2020.

3. Petrosillo N, Viceconte G, Ergonul O, Ippolito G, Petersen E. COVID19, SARS and MERS: are they closely related? Clin Microbiol Infect. 2020;26(6):729-34.

4. European Centre for Disease Control and Prevention. Coronavirus disease 2019 (COVID-19) in the EU/EEA and the UK-ninth update. 2020; 23/04/2020.

5. EURORDIS Rare Diseases Europe. Survey shows detrimental impact of coronavirus on rare disease community [press release]. 2020; 04/05/2020.

6. NORD Rare Insights. COVID-19 community survey report. 2020; 05/05/2020.

7. Gruppo di lavoro ISS Malattie Rare COVID-19. Censimento dei bisogni (23 marzo-5 aprile 2020) delle persone con malattie rare in corso di pandemia da SARS-CoV-2. Istituto Superiore di Sanità. 2020; 30/05/2020.

8. Rare Diseases Ireland. Living with a rare disease in Ireland during the COVID-19 pandemic. 2020; 21/05/2020.

9. European Centre for Disease Control and Prevention. Coronavirus disease 2019 (COVID-19) in the EU/EEA and the UK-eight update. 2020; 08/04/2020.

10. MetabERN. COVID-19 resources. 2020. https://metab.ern-net.eu/covid -19/. Accessed 7 Jul 2020.

11. Sechi A, Macor D, Valent S, Da Riol RM, Zanatta M, Spinelli A, Bianchi K, Bertossi N, Dardis A, Valent F, Scarpa M. Impact of COVID-19 related healthcare crisis on treatments for patients with lysosomal storage disorders, the first Italian experience. Mol Genet Metab. 2020;130:170-1.

12. de Joode K, Dumoulin DW, Engelen V, Bloemendal HJ, Verheij M, van Laarhoven HWM, Dingemans $\mathrm{H}$, Dingemans AC, van der Veldt AAM. Impact of the coronavirus disease 2019 pandemic on cancer treatment: the patients' perspective. Eur J Cancer. 2020;136:132-9.

13. Cancer Research UK. The impact of COVID-19 on cancer patients in the UK. 2020.

14. Joensen LE, Madsen KP, Holm L, Nielsen KA, Rod MH, Petersen AA, Rod $\mathrm{NH}$, Willaing I. Diabetes and COVID-19: psychosocial consequences of the COVID-19 pandemic in people with diabetes in Denmark — what characterizes people with high levels of COVID-19-related worries? Diabet Med. 2020;37:1146-54

\section{Publisher's Note}

Springer Nature remains neutral with regard to jurisdictional claims in published maps and institutional affiliations.

\section{Consent for publication}

Not applicable. 